\title{
Effects of surface irregularities on intensity data from laser scanning: an experimental approach
}

\author{
Arianna Pesci $\left({ }^{1}\right)$ and Giordano Teza $\left({ }^{2}\right)$ \\ (') Istituto Nazionale di Geofisica e Vulcanologia, Sezione di Bologna, Bologna, Italy \\ $\left({ }^{2}\right)$ Dipartimento di Geoscienze, Università di Padova, Padova, Italy
}

\begin{abstract}
The results of an experiment carried out with the aim to investigate the role of surface irregularities on the intensity data provided by a terrestrial laser scanner (TLS) survey are reported here. Depending on surface roughness, the interaction between an electromagnetic wave and microscopic irregularities leads to a Lambertian-like diffusive light reflection, allowing the TLS to receive the backscattered component of the signal. The described experiment consists in a series of TLS-based acquisitions of a rotating artificial target specifically conceived in order to highlight the effects on the intensity data due to surface irregularity. This target is articulated in a flat plate and in an irregular surface, whose macro-roughness has a characteristic length with the same order of the spot size. Results point out the different behavior of the plates. The intensity of the signal backscattered by the planar element decreases if the incidence angle increases, whereas the intensity of the signal backscattered by the irregular surface is almost constant if the incidence angle varies. Since the typical surfaces acquired in a geological/geophysical survey are generally irregular, these results imply that the intensity data can be easily used in order to evaluate the reflectance of the material at the considered wavelength, e.g. for pattern recognition purposes.
\end{abstract}

Key words Terrestrial Laser Scanner - Intensity Incidence angle - Irregular Surfaces - Data Acquisition and Processing.

\section{Introduction}

The time-of-flight terrestrial laser scanner (TLS) provides geometrical and radiometric data on observed objects whose topography can be described with an accuracy of few $\mathrm{cm}$ or better (Rowlands et al., 2003; Pesci et al., 2007; Teza et al., 2007). A TLS works like a laser rangefinder by computing the ranges from the

Mailing address: Dr. Arianna Pesci, Istituto Nazionale di Geofisica e Vulcanologia, Sezione di Bologna, Via Donato Creti, 12, 40128 Bologna, Italy; Tel. +39 051 4151416; Fax +39051 4151498; e-mail: pesci@bo.ingv.it measurement of the time-of-flight of a laser pulse. Moreover, the laser beam is deflected over a calibrated angular grid providing the automatic coverage of a surface in the field of view previously set by the operator. For each spot, the system computes the coordinates of the measured point into an internal reference frame. Each pulse illuminates a small area of the observed surface, with a spot size increasing almost linearly with the range. The present generation of long range laser scanners is characterized by a very collimated laser beam and the spot size is in the order of $2-3 \mathrm{~cm}$ for $100 \mathrm{~m}$ range, linearly increasing to about $10 \mathrm{~cm}$ at $1000 \mathrm{~m}$ range. The TLS is an active instrument with an operational range usually less than 800$1000 \mathrm{~m}$, mainly depending on illuminated material characteristics such as roughness and color (Fidera et. al., 2004). Moreover, instruments with 2000-m limit-range are also available. 
The accuracy of the acquired laser points mainly depends on the observing distance, on the angle of incidence between the beam and the observed surface, and on the quality of the internal telemeter. For example, the Optech ILRIS $^{\mathrm{TM}}$ 3D TLS is able to acquire a single point at $50 \mathrm{~m}$ distance with about $7 \mathrm{~mm}$ accuracy and $17.7 \mathrm{~mm}$ resolution (Lichti and Jamtsho, 2006).

The large amount of acquired data allows the generation of a final model whose accuracy is better than the one on the single point (Geoff, 2005; Fidera et al., 2004). Moreover, a TLS is able to discriminate between the first and the last arrival for each pulse. If the last pulse mode is used, the effects of vegetation or other noises affecting the physical surface modeling (Schulz and Ingerstand, 2004) can be reduced. For these reasons, the TLS can be adopted to observe and model small areas, where high accuracy is needed, to integrate models obtained with different methods (e.g. aerial digital photogrammetry).

An unique feature of this instrumental technique is that no common station points are required in multi-temporal surveys because the results are independently georeferenced, even if the observational protocols must be defined to allow a valid comparison between the corresponding models (Teza et al., 2007).

As stated above, together with the point coordinates, the intensity, i.e. the power of the backscattered radiation, is measured for each acquired point. The material reflectance could be computed, starting from data intensity, and some qualitative and quantitative evaluations could be performed (Kurazume et al., 2002). The intensity data could provide interesting information but it requires suitable investigation aimed to verify their validity in a typical TLSbased geological/geophysical survey. For this reason, an experiment based on the use of an artificial target was performed. The main results and implications of this experiment are reported here.

\section{Intensity acquisition and computation}

The product of a TLS observational session is the point cloud

$$
\left\{\left(x_{k}, y_{k}, z_{k}, I\left(x_{k}, y_{k}, z_{k}\right)\right), k=1,2, \ldots, N\right\}
$$

where $I\left(x_{k}, y_{k}, z_{k}\right)$ is the intensity related to the $k$-th point, i.e. it is a value proportional to the power carried by the received pulse, and $N$ is the number of acquired points (generally, $N$ $\left.10^{5}-10^{6}\right)$. The intensity directly provided by the instrument is called RAW intensity. The ILRIS instrument collects data in two channels providing a hexadecimal (16-bit) digital number (DN). The currently available TLS data processing software packages generally require point clouds of the type

$$
\left\{\left(x_{k}, y_{k}, z_{k}, I_{n}\left(x_{k}, y_{k}, z_{k}\right)\right), k=1,2, \ldots, N_{e}\right\}
$$

where $I_{n}$ is the intensity normalized to the range $[0,255] . I_{n}$ is expressed by an 8 bit DN like a monochromatic image. $N_{e} \cong N$ is the number of points where the intensity can be effectively defined (for example, $I_{n}\left(x_{k}, y_{k}, z_{k}\right)$ is not provided is the signal backscattered by the target at the position $\left(x_{k}, y_{k}, z_{k}\right)$ saturates). The algorithm used to compute $I_{n}$ starting from $I$ takes into account the target distance, magnifies the faint signals to make them well visible in the point cloud, and improves the visual representation of the point cloud. Finally, the values 0 and 255 correspond to the minimum and maximum intensities detected in the entire point cloud after these elaborations. In this way, the points representing objects having the same reflectance at the laser signal wavelength, and acquired in the same scan at different distances, have similar normalized intensities, even if the acquired intensities can be very different. Other TLS manufacturers have developed specific parsing software where other normalization algorithms are implemented. Henceforward, in this article the term intensity will indicate the normalized intensity, i.e. the 8-bit DN, instead of the RAW one (16-bit DN).

A protocol for good quality TLS data acquisition requires that the laser beam incidence should be as normal as possible to the analyzed surface. The probability of backscattering decreases if the angle between the laser beam and the normal to the observed surface increases. If 
this angle is too high, fewer points are acquired and the accuracy decreases.

The backscattered signal power depends on both physical properties of the reflecting material and geometrical condition, that is the relative position of TLS and observed surface (incidence angle). The intensity strongly depends on the material moisture, since a long range TLS generally operates in the near infrared (IR) band (in the case of Optech ILRIS 3d, the wavelength is $1535 \mathrm{~nm}$ ) and the water content of the material can modify the IR reflectance (Kurazume et al., 2002). Therefore, a same object observed in different epochs and meteorological settings, can return different intensities (Mazzarini et al., 2007). Geometry and chemical characteristics are not the only factors affecting the radiometric response but also external ones like humidity and wet conditions. For this reason, only a relative intensity evaluation within the same survey can be considered, whereas multi-temporal observations could provide data that cannot be directly compared with the initial ones. If two areas of a same point cloud are observed under the same conditions, and the corresponding intensities are suitably corrected, these data could be theoretically used in order to discriminate between different materials.

Figure 1 reports an example of the intensity information contained in a point cloud. This point cloud was acquired on October 2006 during a survey to the Vesuvius volcano (Naples,
Italy), considering a mean distance of about $100 \mathrm{~m}$. A calibrated camera was also used in order to provide the RGB image of the observed crater wall. Since the wavelengths are different (RGB: 400-700 nm; TLS: $1535 \mathrm{~nm}$ ), as well as and the corresponding illumination conditions (TLS is an active instruments), RGB and intensity data are substantially independent and could be advantageously integrated to characterize the studied volcanic environment and to detect interesting features like faults and other discontinuities. On the other hand, TLS intensity data can be used in order to study stratigraphic successions (Bellian et al., 2005).

\section{The experiment}

In order to verify the reliability of intensity TLS data for geological/geophysical applications, an experiment was performed in April 2008. The main aim were to verify if the intensity corrections automatically performed by a typical parsing software provide good data for reflectance estimation purposes. The used instrument was Optech ILRIS 3D (Optech, 2008), and the binary data provided by this instrument were parsed using Optech Parser 4.2.7.2 to obtain a point cloud in PIF format processed by Innovmetric PolyWorks 9 (Innovmetric, 2008).

The hypothesis was that the spot spreading on a surface returns a reduced signal to the
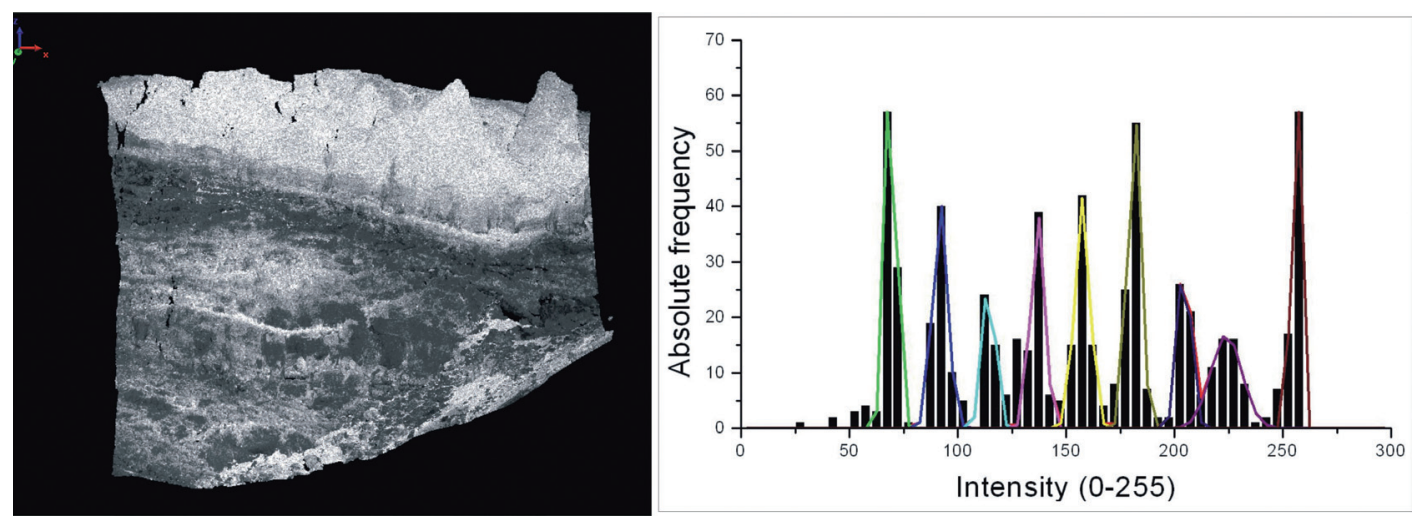

Fig. 1a,b. a) Point cloud of a volcanic wall in the Vesuvius crater acquired from a distance less than $100 \mathrm{~m}$ in October 2006; b) Statistical distribution of intensity values extracted from six vertical profiles of the wall. 
TLS' receiver and the intensity provides an underestimation of the reflectance. This assumption seemed to be reasonable taking into account the surface roughness, the incidence angle, i.e. the angle between the shoot surface and the laser beam, and the material roughness. The latter is responsible for the backscatter quality of an element and refers to its microscopic features (Lichti and Harvey, 2002). In particular, if a vertical planar surface is observed considering an incidence angle $\theta$ and the material is assumed to be perfectly Lambertian, a reduction of the intensity by the factor $\cos \theta$ is expected.

On the other hand, the spot diameter is 2 $\mathrm{cm}$ at $50 \mathrm{~m}$ for a typical long range instrument. Therefore, a question arises about the macroscopic surface irregularities that range from a few $\mathrm{mm}$ to several $\mathrm{cm}$; are they able to neutralize the intensity reduction related to the incidence angle increase? The sense of this question is that in geological/geophysical applications the observed natural surfaces are irregular. For example, if the image of a volcanic wall is zoomed, the fact that the surface consists of several elements like fresh basaltic glass, altered glass, finely crystallized volcanic fragments (e.g. tachylite), crystals, or also volcanic sand/silt, therefore leading to a macroscopic roughness of some $\mathrm{cm}$, appears to be clear.

The experiment was performed using a specifically designed artificial target composed of a flat white panel, able to rotate on its central axis, and of two dark painted units mounted on it (fig. 2). The first unit was built using several small elements of pasta (i.e. alimentary paste) with different shapes and sizes (rice, macaroni, and others), to materialize a surface characterized by known irregularities ranging from a few millimeters to some centimeters. The second unit was a simple flat rectangle. Moreover, the white panel supporting the two units was anchored to an external frame made of the same material.

The white rectangular frame provided a reference with a well defined shape and reflectance. The rotating plate was acquired by the TLS at different incidence angles between $0^{\circ}$ and $75^{\circ}$, with $15^{\circ}$ angular step. Obviously, $0^{\circ}$ corresponds to normal incidence of the laser beam with respect to the target.
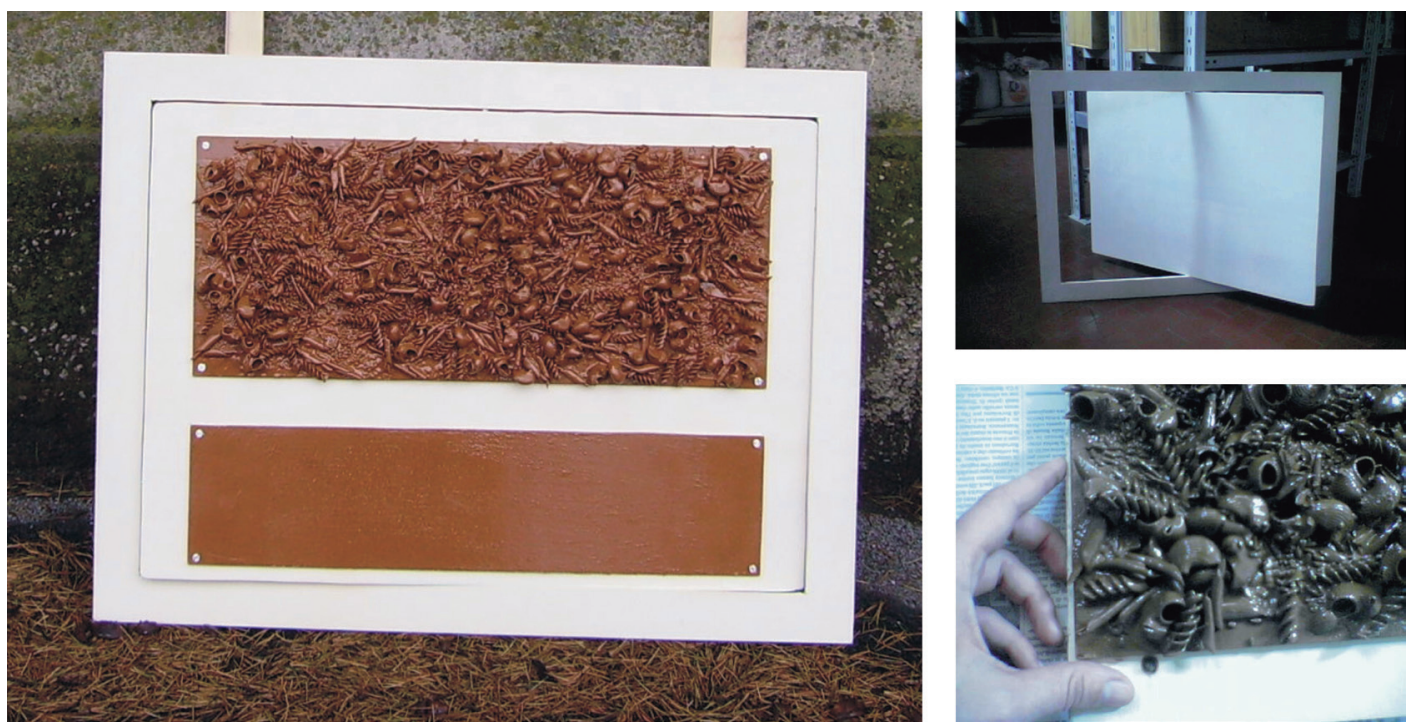

Fig. 2. Rotating target. The irregular surface of the upper module mounted on the white panel has a 1-cm mean length. 
At first, the target was vertically stabilized and scanned from a distance of about $25 \mathrm{~m}$. Six scans were performed according to the above described incidence angles. The six point clouds are shown in fig. 3. The intensity data belonging to the target components were extracted from each of the six point clouds computing the mean 8-bit intensity values, listed in table I. The data acquisition was repeated using the same angles and the same observational protocol at distances $50 \mathrm{~m}, 100 \mathrm{~m}$, and $225 \mathrm{~m}$. Therefore, the complete experiment was articulated on acquisition and analysis of 24 point clouds. Since no significant differences appear when different distances are considered, only the re- sults of the $25 \mathrm{~m}$ and $50 \mathrm{~m}$ cases are presented here.

Clearly, the white frame intensities are stable at the same 255 value, while the ones related to the points belonging to the white rotating panel and the dark unit placed on it decrease when the incidence angle increase, according to the expected behavior because the reflectors are regular surfaces with respect to the footprint size (fig. 4). For each element constituting the target (that is the white frame, the white plate, the dark flat and irregular units) the intensity data was extracted and listed in table I.

Note that the value 255 does not mean saturation, but the maximum intensity detected in
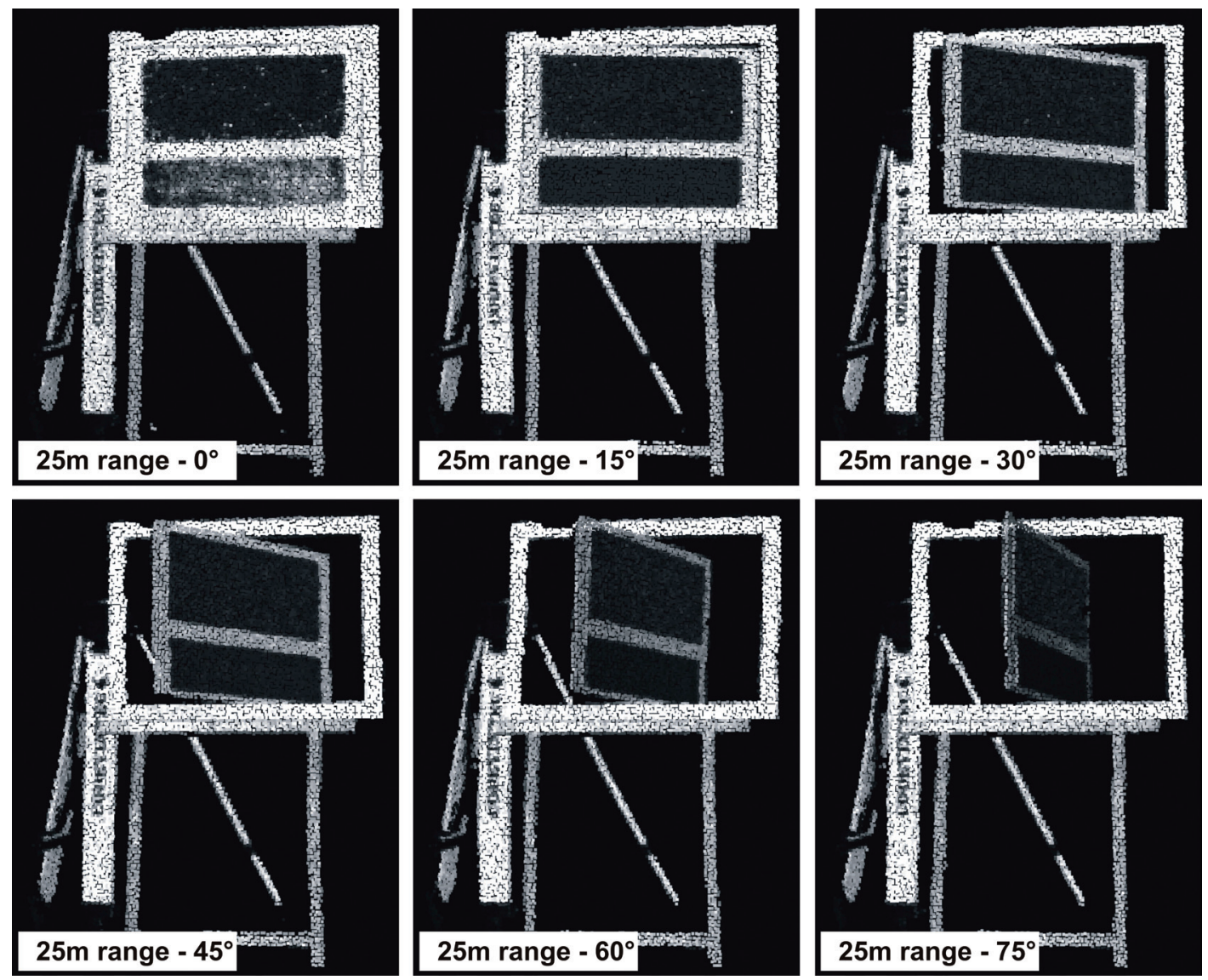

Fig. 3. Six scans acquired from $25 \mathrm{~m}$ distance at different rotation angle. 
Table I. Results from the first acquisition at $25 \mathrm{~m}$ distance; four mean intensity values are listed for each position concerning the parts of the target.

Scans from $25 \mathrm{~m}$ distance

\begin{tabular}{ccccc}
\hline Angle $\left(^{\circ}\right)$ & White frame & White plate & Dark flat unit & Dark irregular unit \\
\hline 0 & 255 & 246 & 50 & 23 \\
15 & 255 & 227 & 28 & 24 \\
30 & 255 & 194 & 25 & 23 \\
45 & 255 & 163 & 21 & 22 \\
60 & 255 & 118 & 15 & 21 \\
75 & 255 & 60 & 7 & 20 \\
\hline
\end{tabular}

the point cloud. As already reported, Optech Parser excludes the points whose backscattered pulses saturate the detector. Linear interpolations are considered in order to evaluate $I_{n}=I_{n}(\theta)$, where $\theta$ is the incidence angle (fig. 5).
Although the intensity decays can be fitted by a parabolic or also a linear law for both the white rotating panel and the dark unit, the corresponding coefficients are quite different, i.e. the intensity reduction is significantly stronger in

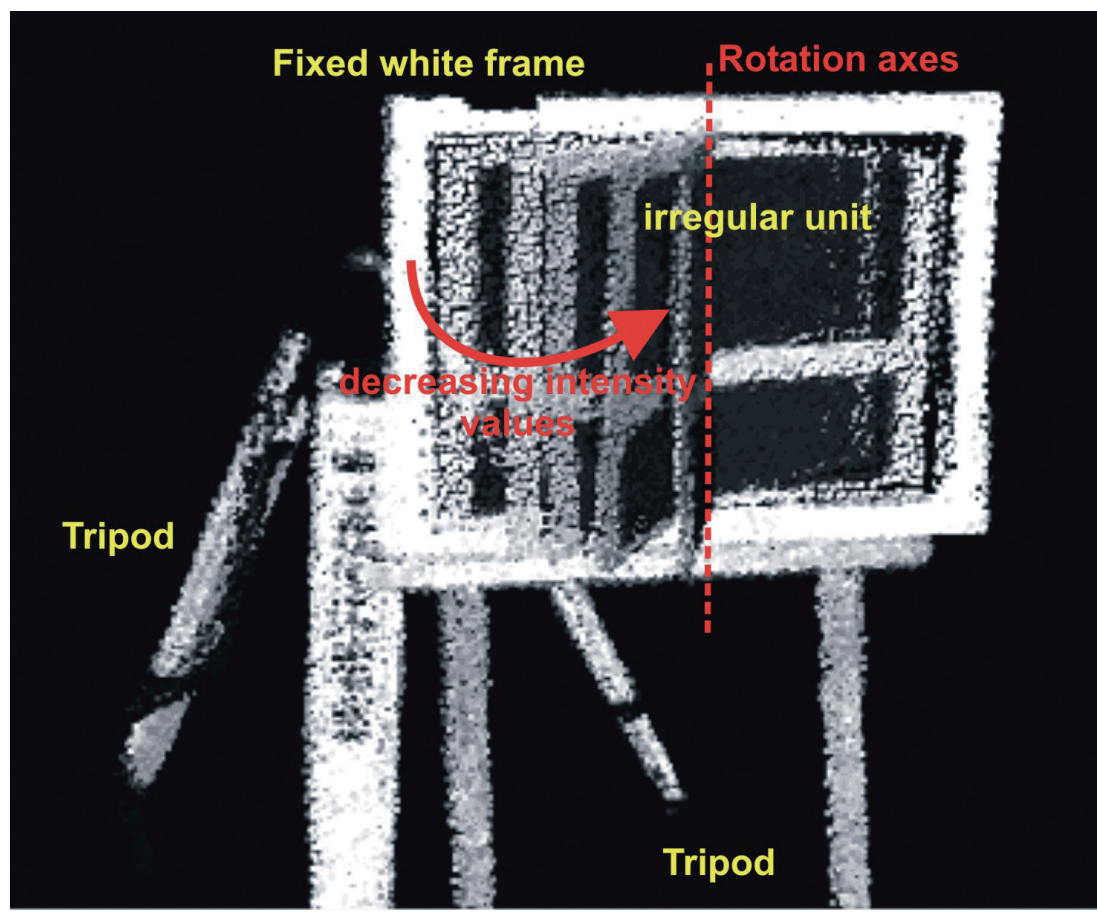

Fig. 5. Intensity data and linear trend interpolation of the white panel and dark flat unit from the $25 \mathrm{~m}$ range scans. The intensities rapidly decrease with the incident angle whereas the frame and the irregular surface lead to almost constant intensities. Note that the histograms are strongly picked. 
the case of the most reflective material. The first order polynomials obtained via least square fits are $I_{n}$, WHITE $=260-25 \theta$ and $I_{n}$, DARK $=35-0.35 \theta$ respectively, where intensities are expressed as $\mathrm{DN}$, as usual. A scale factor seems to relate the two linear trends, since the ratio between the same degree coefficients are very similar, i.e. 7.5 (zero degree coefficients) and 7.1 (first degree).

The intensity corresponding to the irregular dark unit, on the contrary, is almost the same if the incidence angle changes. This means that the backscattered signal is not sensitive to the relative position between the instrument and the observed surface. In other words, the laser pulses are diffused on all the half-space without any preferential direction because a large amount of macroscopic elements are always normal to the incident laser beam. All these results are qualitatively summarized in fig. 4, which highlights the high variation rate of the white panel intensity and a very low variation rate for the dark plate.

The same analysis was performed using data at other distances; the results obtained at $50 \mathrm{~m}$ are listed in table II and reported in fig. 6 . The first order polynomials obtained via least square fits are now $I_{n}$, WHITE $=254-2.7 \theta$ and $I_{n}, D A R K=36-0.41 \theta$. The corresponding coefficient ratios are 7.1 and 6.8 for the zero and fist degree term respectively, like the 25 -m case.

The experiment showed that the higher the angle the lower the intensity, and this fact seems to be reasonable because the spot spread- ing causes energy dispersion with respect to the Lambertian nature of the physical surface. Nevertheless, a question concerning the different rates for intensity decay emerged; the existence of a scale factor between the linear fits representing the function $I_{n}=I_{n}(\theta)$ for high and low reflectance flat materials required a better investigation. Since the angle-dependent decay was a significantly higher velocity in the case of the most reflective unit, a verification that this fact really depends on the involved material and is not an artifact due to the parsing software, seemed to be strictly necessary. For this reason, the normalized intensities were compared with the RAW ones, as depicted in fig. 7. This figure emphasizes that the 8-bit DN and 16-bit DN show similar features, even if the corresponding intensity ranges are different, i.e. $[0,255]$ and $[0,5000]$ respectively. Moreover, the numerical step seems to be different based on the measured level, showing a different and not constant scale factor. This fact could be related to the effect of high gain and low gain channels of the received pulse detector. Figure 8 shows the statistical distribution of the RAW intensities extracted from the $25 \mathrm{~m}-15^{\circ}$ and the $25 \mathrm{~m}-30^{\circ}$ point clouds, highlighting a significant value reduction.

The approach that was previously applied to evaluate $I_{n}=I_{n}(\theta)$ was also used to study the angle-dependence of the RAW intensity for different flat surfaces placed at the same distance from the instrument. Also in this case

Table II. Results of the data acquisition at $50 \mathrm{~m}$ distance. Four mean intensity values are listed for each position concerning the parts of the target.

Scans from $50 \mathrm{~m}$ distance

\begin{tabular}{ccccc}
\hline Angle $\left({ }^{\circ}\right)$ & White frame & White plate & Dark flat unit & Dark irregular unit \\
\hline 0 & 249 & 247 & 86 & 25 \\
15 & 250 & 218 & 30 & 25 \\
30 & 249 & 177 & 23 & 24 \\
45 & 248 & 132 & 20 & 24 \\
60 & 243 & 81 & 12 & 21 \\
75 & 248 & 50 & 5 & 22 \\
\hline
\end{tabular}




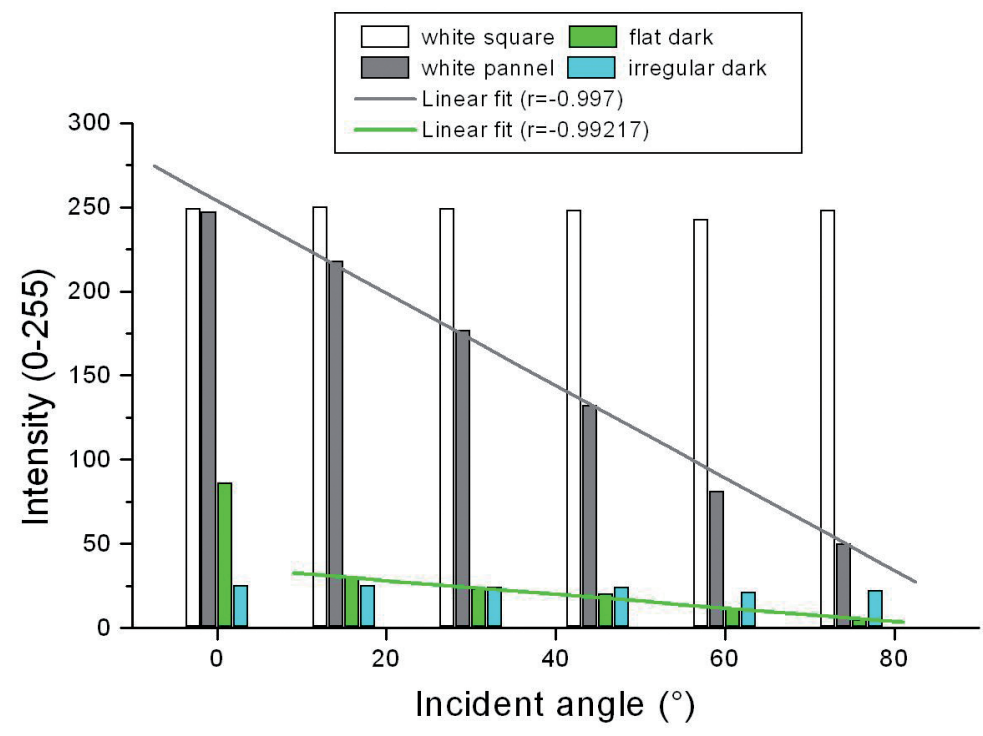

Fig. 6. Intensity data and linear trend interpolation of the white panel and dark flat unit from the $50 \mathrm{~m}$ range scans. The intensities rapidly decrease with the incident angle whereas the frame and the irregular surface lead to almost constant intensities. Note that the histograms are strongly picked.

different decay rates were detected. This fact demonstrated that, inside a same point cloud, the observed behaviors primarily depend on the reflectance of the observed material, and that the normalized intensity could therefore be used for material recognition purposes. On the other hand, if different point clouds are considered, an intensity-based material recognition could lead to some difficulties because the normalization also involves the minimum and minimum detected intensities. In such a case, a RAW intensity-based approach should be considered.

\section{Discussion and conclusions}

The analysis of the point clouds acquired from a $25 \mathrm{~m}$ range pointed out that the intensity responses of different materials were very different. The returned intensities of the rotating white panel and of the dark plate decreased with the rotation angle. On the contrary, the irregular dark unit lead to an almost low constant value. The same effects were observed in scans from longer distances $(50 \mathrm{~m}, 100 \mathrm{~m}$ and $250 \mathrm{~m})$.

Since the flat regular elements only was affected by intensity reduction, whereas no significant effects characterized the intensity of the irregular unit, a possible interpretation is related to the macroscopic irregularity level of the observed objects. Here, the term «irregular»means «irregular with respect to the laser footprint», i.e. the surface consists of variously oriented elements whose characteristic size does not exceed the footprint. For example, for a $3 \mathrm{~cm}$ footprint, a surface is irregular if the irregularities are in the range $\sim 1 \mathrm{~mm}-\sim 1 \mathrm{~cm}$, whereas a painted surface with $\sim 0.1 \mathrm{~mm}$ size irregularities can be considered regular from this viewpoint. It was the case of the flat unit. If a surface is irregular, it can therefore be considered a Lambertian, constant target.

A comparison between the 8 bit DN, i.e. normalized intensity, and 16 bit DN, i.e. RAW intensity, of a same point cloud showed that the observed behaviors are related to the material reflectance (at the observational conditions, since the reflectance depends, e.g., on moisture) 


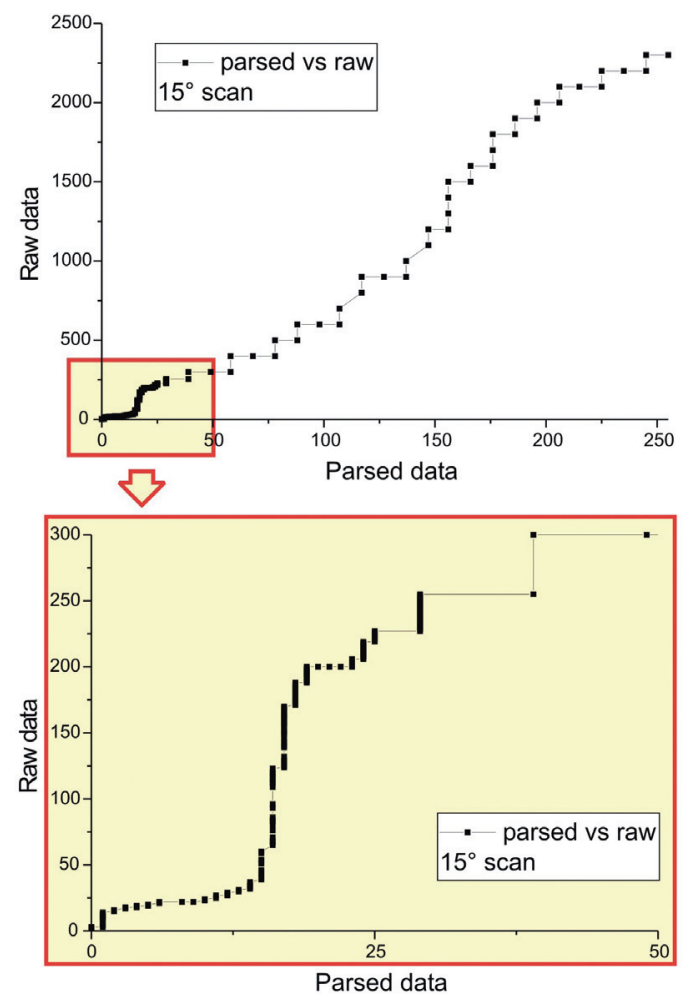

Fig. 7. (new). RAW and normalized intensity data extracted from the same scan at $25 \mathrm{~m}$ range and at $15^{\circ}$ incidence. and are not artifacts caused by the parsing software. In particular, if a regular surface is considered, the higher is the rotation angle, the lower is the intensity of the returned pulse and, moreover, the rate of the angular decay increases with the intensity measured at normal incidence.

In summary, an analysis of the features that can be recognized in a point cloud on the basis of the material reflectance can be easily performed if the material irregularities (macroscopic roughness) have a characteristic size that does not exceed the laser footprint diameter. In that case, the distance correction performed by the parsing software is enough for recognition purposes inside a point cloud. Note that the mentioned condition on macro-roughness is generally satisfied dealing with natural surfaces. The components of a stratigraphic succession should be therefore easily recognized if no human artificial works (like surface smoothing) were performed on the observed surface. Moreover, good intensity levels, also in conditions far from the normal incidence, imply that the mean accuracy in data acquisition is about constant in all the region of the observed surface. Nevertheless, the intensity analysis is much more complicated if the observed surface is roughly planar with respect the footprint, which is the typical case of the TLS data acquisition

(a)
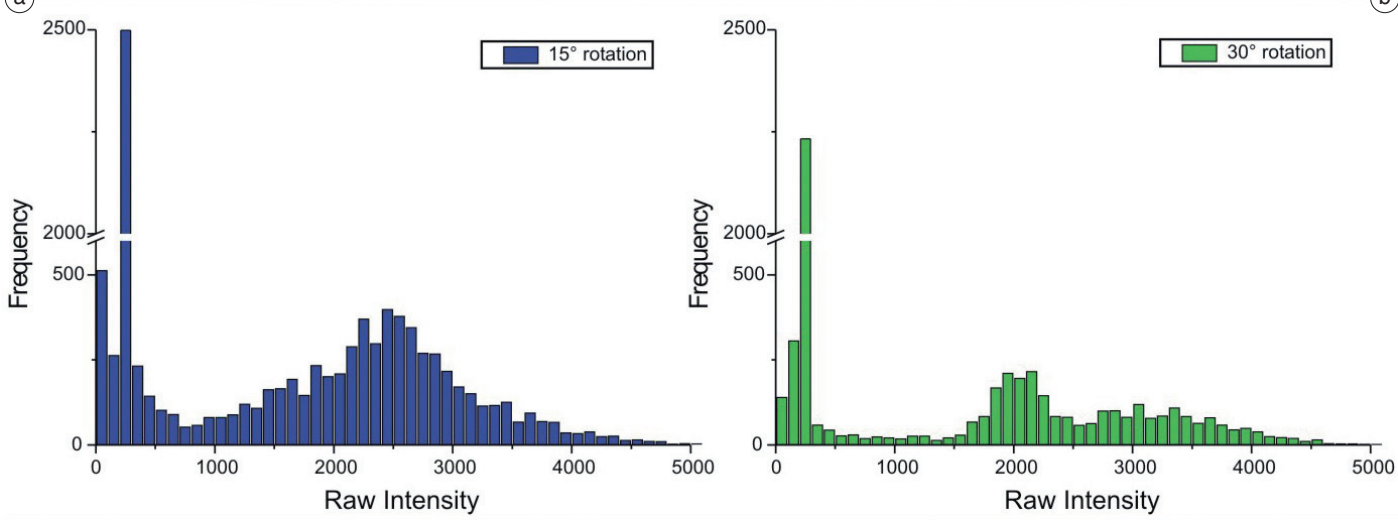

Fig. 8. Statistical distributions of RAW intensity data for $15^{\circ}$ (a) and $30^{\circ}$ (b) incidence angle respectively. 
for architectural or cultural heritage purposes. In addiction, some problems arise if the aim is the comparison between data related to different point clouds. For these reasons, a study of the relation between the material reflectance and the RAW DN will be the subject of a future work.

\section{Acknowledgment}

The authors thank Marco Bacciocchi and Andrea Faccioli for instrumental support and Massimo Bacchetti for the valuable assistance in target creation.

\section{REFERENCES}

Bellian, J.A., C. Kerans and D.C. Jannette (2005): Digital Outcrop Models: application of terrestrial scanning LIDAR technology in stratigraphic modeling, Journal of Sedimentary Research, 75 (2), 166-176.

FIDERA, A., M.A. CHAPMAN and J. HONG (2004): Terrestrial Lidar for industrial metrology applications: modeling, enhancement and reconstruction, in Proceedings of XXth ISPRS congress, (Istanbul, Turkey, 12-23 July 2004), 880-883.

GEOFF, J. (2005): Understanding laser scanner terminology, Professional Surveyor Magazine, 25 (2), 22-28. Available online at: http://www.profsurv.com/archive.php? issue $=97 \&$ article $=1377$ (accessed: 07.05.08).

INNOVMETRIC (2008): PolyWorks software package technical data. Available online at: http://www.innovmetric. com/Manufacturing/home.aspx (accessed: 07.07.08).

Kurazume, R., K. Nishino, Z. Zhang and K. IKeuchi (2002): Simultaneous 2D images and 3D geometric model registration for texture mapping utilizing reflectance attribute, in Proceedings of the 5th Asian
Conference on Computer Vision (ACCV), (Melbourne, Australia, January 22-25, 2002), 99-106.

LITCHI, D. and B. HARVEY (2002): The effects of reflecting surface material properties on time-of-flight laser scanner measurements, in Proceedings of Symposium of Geospatial theory, processing and applications, (Ottawa, Canada, July 9-12, 2002). Available online at: http://www.isprs.org/commission4/proceedings02/pdfpapers/180.pdf (accessed: 07.05.08).

LichTI, D. and S. JAMTSHO (2006): Angular resolution of terrestrial laser scanners, The Photogrammetric Record, 21 (114), 141-160.

Mazzarini F., M.T. Pareschi, M. Favalli, I. Isola, S. TARQUINI and E. BosCHI (2007): Lava flow identification and ageing by means of LiDAR intensity: the Mt. Etna case, Journal of Geophysical Research, 112, B02201, doi: 10.1029/2005JB004166.).

OPTECH (2008): Laser scanner ILRIS 3D technical data. Available online at: http://www.optech.ca/i3dtechoverview-ilris.htm (accessed: 07.05.2008).

Pesci, A., F. Loddo and D. Conforti (2007): The first terrestrial laser scanner application over Vesuvius (Napoli, Italy): the high resolution model of volcano crater, International Journal of Remote Sensing, 28 (1-2), 203-219.

ROWLANDS K.A., L.D. JONES and M. WHITWORTH (2003): Landslide Laser Scanning: a new look at an old problem, Quarterly Journal of Engineering Geology and Hydrogeology, 36, 155-157.

Schulz, T. and M. INGENSAND (2004): Influencing variables, precision and accuracy of terrestrial laser scanners, in Proceedings of INGEO 2004 and FIG Regional Central and Eastern European Conference of Engineering Surveying, (Bratislava, Slovakia, November 11-13, 2004), Available online at: http://www-roup. slac.stanford.edu/met/Align/Laser_Scanner/SchulzT_ TS2_Bratislava_2004.pdf (accessed: 07.05.08)

Teza, G., A. Galgaro, N. Zaltron and R. Genevois (2007): Terrestrial laser scanner to detect landslide displacement fields: a new approach. International Journal of Remote Sensing, 28 (16), 3425-3446.

(received May 9, 2008; accepted June 12, 2008) 